\title{
Vagus nerve stimulation attenuates myocardial ischemia/reperfusion injury by inhibiting the expression of interleukin-17A
}

\author{
CHUNFENG $\mathrm{YI}^{1}$, CHANGJIANG ZHANG ${ }^{2}$, XIAORONG HU ${ }^{1}$, YUANHONG LI ${ }^{2}$, HONG JIANG ${ }^{1}$, \\ WEIPAN XU ${ }^{1}$, JIAJIA LU ${ }^{1}$, YUANXI LIAO ${ }^{1}$, RUISONG MA ${ }^{1}$, XUEFEI LI ${ }^{1}$ and JICHUN WANG ${ }^{1}$ \\ ${ }^{1}$ Department of Cardiology, Renmin Hospital of Wuhan University, Cardiovascular Research Institute of Wuhan University, \\ Wuhan, Hubei 430060; ${ }^{2}$ Department of Cardiology, Central Hospital of Enshi Tujia and Miao Autonomous Prefecture, \\ Enshi, Hubei 445000, P.R. China
}

Received January 28, 2015; Accepted October 21, 2015

DOI: $10.3892 /$ etm.2015.2880

\begin{abstract}
Interleukin (IL)-17A has an important role in myocardial ischemia/reperfusion (I/R) injury, and vagal stimulation (VS) has been demonstrated to exert cardioprotective effects. The present study aimed to investigate the effects of VS on a rat model of myocardial I/R injury, and detected an association between VS and IL-17A. Anesthetized rats underwent VS (2 msec; $10 \mathrm{~Hz})$ or were treated with anti-IL-17A neutralized monoclonal antibodies (mAbs) $(200 \mu \mathrm{g}$; iv), and subjected to ischemia for $30 \mathrm{~min}$ prior to $4 \mathrm{~h}$ reperfusion. The following parameters were measured: Infarct size; lactate dehydrogenase (LDH), creatine kinase (CK), malondialdehyde (MDA), superoxide dismutase (SOD) and caspase-3 activity levels; tumor necrosis factor (TNF)- $\alpha$ and IL-6 expression levels; and the percentage of terminal deoxynucleotidyl-transferase mediated dUTP nick-end labeling (TUNEL) positive cells . High mobility group box 1 protein (HMGB1) and IL-17A expression levels were assessed by immunoblotting. Following $4 \mathrm{~h}$ reperfusion, VS was able to significantly decrease the infarct size and the activity levels of LDH and CK $(\mathrm{P}<0.05)$. Furthermore, VS administration significantly suppressed the increased MDA and decreased SOD activity levels, and significantly reduced caspase- 3 activity and the percentage of TUNEL-positive cells $(\mathrm{P}<0.05)$. Treatment with anti-IL-17A mAbs demonstrated the same effects as VS. Furthermore, VS was able to significantly inhibit the increased expression levels of TNF- $\alpha$, IL-6, HMGB1 and IL-17A induced by I/R $(\mathrm{P}<0.05)$. The results of the present study suggested that VS may attenuate myocardial I/R injury by reducing the expression of
\end{abstract}

Correspondence to: Mr. Yuanhong Li, Department of Cardiology, Central Hospital of Enshi Tujia and Miao Autonomous Prefecture, 158 Wuyang Road, Enshi, Hubei 445000, P.R China

E-mail: yuanhongwhu@126.com

Key words: vagal stimulation, interleukin-17A, inflammation, myocardial ischemia, reperfusion inflammatory cytokines, oxidative stress and the apoptosis of cardiomyocytes. Furthermore, VS may induce cardioprotective effects, which may be associated with the inhibition of IL-17A expression.

\section{Introduction}

Myocardial reperfusion is a therapeutic strategy which is commonly used to treat patients with acute coronary syndrome; however, reperfusion following ischemia may promote additional cellular injury, which accounts for $\sim 50 \%$ of the final size of a myocardial infarct (1). A previous study demonstrated that ischemia/reperfusion (I/R) may promote the production of numerous proinflammatory cytokines, neutrophil infiltration and the apoptosis of cardiomyocytes, which is likely to result in cardiomyocyte damage (1). Interleukin (IL)-17A, which is a member of the IL-17 cytokine family, is secreted by CD4 ${ }^{+}$ $\alpha \beta$ T cells, ro T cells, natural killer cells, and neutrophils (2). Previous studies have demonstrated that IL-17A may stimulate the production of numerous proinflammatory mediators, including cytokines, chemokines and matrix metalloproteinases (3-5). It has been demonstrated that IL-17A is primarily produced by $\mathrm{r} \delta \mathrm{T}$ cells following myocardial $\mathrm{I} / \mathrm{R}$ injury, and IL-17A has a pathogenic role by inducing neutrophil infiltration and the apoptosis of cardiomyocytes (6). Therefore, IL-17A may facilitate the release of proinflammatory cytokines and thus may have a key role in myocardial I/R injury.

Previous studies have demonstrated that vagal stimulation (VS) and acetylcholine (ACh) are capable of exerting cardioprotective effects in various cardiovascular diseases $(7,8)$. Furthermore, the inhibition of inflammation has been demonstrated to be one of the major pathophysiological mechanisms by which VS induces cardioprotection $(9,10)$. As a proinflammatory cytokine, IL-17A interacts with other proinflammatory mediators, including tumor necrosis factor (TNF)- $\alpha$, IL-1 $\beta$ and IL-6 (11); therefore, VS and IL-17A may be associated with the regulation of apoptosis in cardiomyocytes $(6,12)$. The authors of the present study hypothesized that there may be a cardioprotective association between IL-17A and VS. In the present study, the hypothesis that VS may attenuate 
myocardial I/R injury by suppressing IL-17A expression levels was investigated in a rat model of myocardial I/R.

\section{Materials and methods}

Animal preparation andexperimental design. The present study conformed to the Guideline for the Care and Use of Laboratory Animals published by the US National Institutes of Health (NIH Publication, revised 1996) (13) and was approved by the Renmin Hospital of Wuhan University Animal Care and Use Committee (Wuhan, China). A total of 48 male Sprague-Dawley rats, weighing 250-300 g, were supplied by the Experimental Animal Center of Vital River Laboratories (Beijing, China). The rats were randomized into four equal groups, as follows: Group 1, sham operated (SO) rats were subjected to surgical manipulation without the induction of myocardial ischemia ( $\mathrm{n}=12)$; group $2, \mathrm{I} / \mathrm{R}$ rats were subjected to occlusion of the left anterior descending coronary artery (LAD) for $30 \mathrm{~min}$, followed by reperfusion for $4 \mathrm{~h}(\mathrm{n}=12)$; group $3, \mathrm{I} / \mathrm{R}+\mathrm{VS}(\mathrm{n}=12)$ rats were treated the same as group 2 , with the exception that VS was initiated $15 \mathrm{~min}$ post-ischemia and lasted for $30 \mathrm{~min}(9)$; and group 4, I/R + anti-IL-17A $(\mathrm{n}=12)$ rats were treated the same as group 2, with the exception that $200 \mu \mathrm{g}$ anti-IL-17A neutralized monoclonal antibodies (mAbs) (Biosynthesis Biotechnology Co., Ltd., Beijing, China) were intravenously injected 30 min prior to occlusion of the LAD (4).

The rats were anesthetized with sodium pentobarbital ( $45 \mathrm{mg} / \mathrm{kg}$, i.p.), fixed on an electric heating pad to maintain their body temperature at $37^{\circ} \mathrm{C}$, and subsequently artificially ventilated with a volume-controlled rodent respirator at 70 strokes/minute. Lead II of the electrocardiogram was recorded using subcutaneous stainless steel electrodes. The electrocardiogram was monitored during operation and vagal stimulation using a biological signal recording system (MP150; BIOPAC Systems, Inc., Goleta, CA, USA)

In the SO, I/R and anti-IL-17A groups, rats underwent right cervical vagus nerve trunk exposure without stimulation. In the VS group, the right cervical vagus nerve trunk was isolated from the surrounding tissue with a small piece of parafilm. A pair of bipolar platinum electrodes, covered with warmed paraffin oil for insulation, was placed on the vagus nerve trunk and the vagal nerve was subsequently stimulated by rectangular electrical pulses at $10 \mathrm{~Hz}$ for $2 \mathrm{msec}$ using a S88 Nerve and Muscle Stimulator (Grass Technologies; Natus Neurology Inc., Warwick, RI, USA). The electrical voltages of the pulses were optimized in each rat during stimulation in order to obtain a 10\% reduction in heart rate from the baseline values. The actual stimulus voltage was 1-7 V.

The rats underwent a 30-min LAD occlusion, followed by reperfusion for $4 \mathrm{~h}$ (14). Briefly, a left thoracotomy and pericardiectomy were performed in order to expose the anterior wall of the left ventricle. Using a small curved needle, a 5-0 silk suture was subsequently passed through the myocardium beneath the middle segment of the LAD branch coursing down the middle of the anterior wall of the left ventricle. A small vinyl flake was passed into the ends of the suture, which was subsequently fixed by clamping the tube with a mosquito haemostat. Following this, the chest was closed under negative pressure. Successful occlusion of the LAD was confirmed by alterations in the elevation of the ST segment in Lead II and regional cyanosis of the myocardial surface.

Assessment of infarct size. Following $4 \mathrm{~h}$ reperfusion, the LAD was occluded for a second time and $2 \mathrm{ml}$ Evans Blue Dye (1\%; Sigma-Aldrich, St. Louis, MO, USA) was subsequently injected via the jugular vein. The rats were sacrificed by cardiac puncture under anesthesia with sodium pentobarbital ( $45 \mathrm{mg} / \mathrm{kg}$, i.p.). Following this, the rat hearts were excised and frozen for $15 \mathrm{~min}$ prior to harvesting of the atria and right ventricles. The left ventricles were cut into transverse sections (thickness, $2 \mathrm{~mm}$ ) from the apex to the base. Subsequently, the risk areas were separated from the colored nonischemic areas (blue), and the slices were incubated with a $1 \%$ solution of 2,3,5-triphenyltetrazolium chloride in $0.2 \mathrm{M}$ Tris buffer $(\mathrm{pH}$ 7.4) stain for $15 \mathrm{~min}$ at $37^{\circ} \mathrm{C}$. The respective infarct sizes (white) and risk areas (red and white) of the sections were determined using an Image-Pro Plus 3.0 image analyzer (Media Cybernetics, Inc., Rockville, MD, USA). Infarct size was expressed as a percentage of the risk area volume $(\%=$ infarct size/risk area) (14).

Assessment of myocardial injury. Following $4 \mathrm{~h}$ reperfusion, blood samples were collected in order to assess the levels of lactate dehydrogenase (LDH) and creatine kinase (CK). Following a 10-min rest, blood samples were centrifuged at $875 \mathrm{x}$ g for $15 \mathrm{~min}$. Prior to analyses; the supernatants were collected and stored at $-80^{\circ} \mathrm{C}$. Analyses of the LDH and CK levels were completed using commercially available LDH and CK Assay kits and standard techniques, according to the manufacturer's protocol (Nanjing Jiancheng Bioengineering Institute, Nanjing, China). Data are expressed in international units (IU)/liter (1).

Assessment of myocardial inflammatory parameters. The expression levels of TNF- $\alpha$ and IL- 6 in the myocardial tissue supernatants were measured using a commercial enzyme-linked immunosorbent TNF- $\alpha$ assay kit (Nanjing Jiancheng Bioengineering Institute), according to the manufacturer's protocol. The sensitivity of the assay was $1 \mathrm{pg} / \mathrm{ml}$ for TNF- $\alpha$ and IL-6.

Cardiac tissue samples were lysed in radioimmunoprecipitation assay lysis buffer (Beyotime Institute of Biotechnology, Haimen, China) for western blotting. Protein concentration was determined using Pierce Bicinchoninic Acid Protein Assay kit (Pierce Biotchnology Inc., Rockville, MD, USA). Protein extracts $(50 \mu \mathrm{g})$ were separated by $10 \%$ sodium dodecyl sulfate-polyacrylamide gel electrophoresis, transferred to polyvinylidene difluoride (PVDF) membranes (EMD Millipore, Billerica, MA, USA) and probed with HMGB1 (1:800; cat. no. BA4277; Wuhan Boster Biological Technology Co., Ltd., Wuhan, China), IL-17A (1:800; cat. no. 13838; Cell Signaling Technology, Inc., Danvers, MA, USA) and $\beta$-actin antibodies (1:800; cat. no. BM0627; Wuhan Boster Biological Technology Co., Ltd.). Following incubation with horseradish peroxidase-conjugated goat anti-mouse (cat. no. BA1051), goat anti-rabbit (cat. no. BA1054) and rabbit anti-goat (cat. no. BA1060) secondary antibodies (1:50,000; Wuhan Boster Biological Technology Co., Ltd.) for $1 \mathrm{~h}$ at room temperature, the membranes were treated with enhanced chemiluminescence reagents (Thermo Fisher Scientific, Inc., 
Waltham, MA, USA) prior to visualization using a FluorChem E imager (ProteinSimple, San Jose, CA, USA). The specific protein expression levels were normalized to the levels of $\beta$-actin on the same PVDF membrane. The absorbance values of each target protein/ $\beta$-actin were used to indicate the relative expression levels of each target protein.

Assessment of myocardial oxidative stress. The activity levels of malondialdehyde (MDA), superoxide dismutase (SOD), oxygen free radicals and lipid superoxides in the myocardium were measured using commercially available MDA and SOD Assay kits (Nanjing Jiancheng Bioengineering Institute), according to the manufacturer's protocol.

Assessment of myocardial apoptosis. In order to detect the apoptosis of cardiomyocytes, terminal deoxynucleotidyl-transferase mediated dUTP nick-end labeling (TUNEL) staining and caspase-3 activity level analyses were performed as previously described (3). The rat hearts were fixed in $4 \%$ paraformaldehyde, embedded in paraffin, cut into $5 \mu \mathrm{m}$ sections and treated as outlined in the manufacturer's protocol for the In Situ Cell Death Detection kit (Roche Diagnostics GmbH, Mannheim, Germany). Brown staining in the nucleus or cytoplasm indicated the cells were TUNEL-positive, and thus apoptotic. In order to determine the percentage of apoptotic cells, the apoptotic cells were counted under a microscope (BX53; Olympus Corporation, Tokyo, Japan) and the results from 10 fields of each group were subsequently calculated.

Caspase-3 activity was measured using a caspase-3 enzyme-linked immunosorbent assay kit, according to the manufacturer's protocol (Beyotime Institute of Biotechnology). The absorbance of p-nitroaniline, which is cleaved by caspase-3, was measured at $405 \mathrm{~nm}$ using a microplate reader (ELx800; Bio-Tek Instruments, Winooski, VT, USA).

Statistical analysis. Unpaired Student's t-tests were used for between-group comparisons; whereas a one-way analysis of variance or Welch t-tests were used for comparisons among groups and a Student-Neuman-Keuls or Dunnett T3 was used for post-hoc multiple comparisons. Data are presented as the mean \pm standard deviation. All analyses were completed using SPSS 17.0 software (SPSS, Inc., Chicago, IL, USA). P $<0.05$ was considered to indicate a statistically significant difference.

\section{Results}

Infarct size. No evidence of myocardial infarction was detected in the SO group, due to a lack of myocardial ischemia induction. The infarct sizes (\%) in the VS and anti-IL-17A groups were significantly decreased, as compared with the I/R group $(\mathrm{P}<0.05 ;$ Fig. 1$)$

LDH and CK activity levels. Following $4 \mathrm{~h}$ reperfusion, the activity levels of $\mathrm{LDH}$ and $\mathrm{CK}$ in the I/R group were significantly increased, as compared with those in the SO group. Furthermore, VS or administration of anti-IL-17A significantly inhibited the increase of LDH and CK levels ( $\mathrm{P}<0.05$; Fig. 2)

TNF- $\alpha, I L-6, I L-17 A$ and HMGB1 expression levels. TNF- $\alpha$ and IL-6 levels in the I/R group were significantly increased,

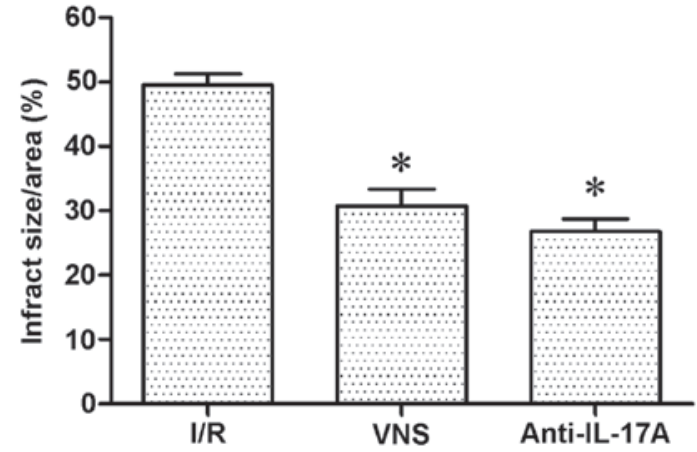

Figure 1. Effects of VNS and anti-IL-17A treatment on infarct size during $\mathrm{I} / \mathrm{R}$ ( $\mathrm{n}=12$ per group). Data are presented as the mean \pm standard deviation. ${ }^{*} \mathrm{P}<0.05$ vs. the I/R group. VNS, vagal nerve stimulation; anti-IL-17A, anti-interleukin-17A monoclonal antibodies; I/R, ischemia/reperfusion.

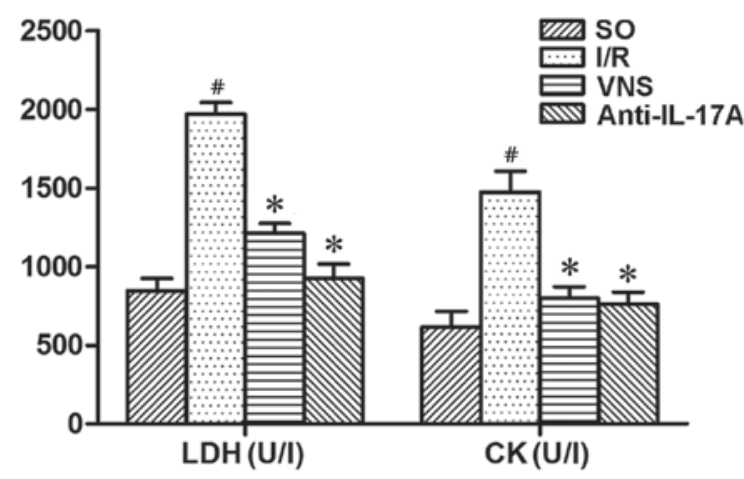

Figure 2. Effects of VNS and anti-IL-17A treatment on LDH and CK activity levels during $\mathrm{I} / \mathrm{R}$ ( $\mathrm{n}=12$ per group). Data are presented as the mean \pm standard deviation. ${ }^{*} \mathrm{P}<0.05$ vs. the $\mathrm{I} / \mathrm{R}$ group; ${ }^{\text {"}} \mathrm{P}<0.05$ vs. the $\mathrm{SO}$ group. VNS, vagal nerve stimulation; I/R, ischemia/reperfusion; anti-IL-17A, anti-interleukin-17A monoclonal antibodies; SO, sham operated control; LDH, lactate dehydrogenase; CK, creatine kinase.

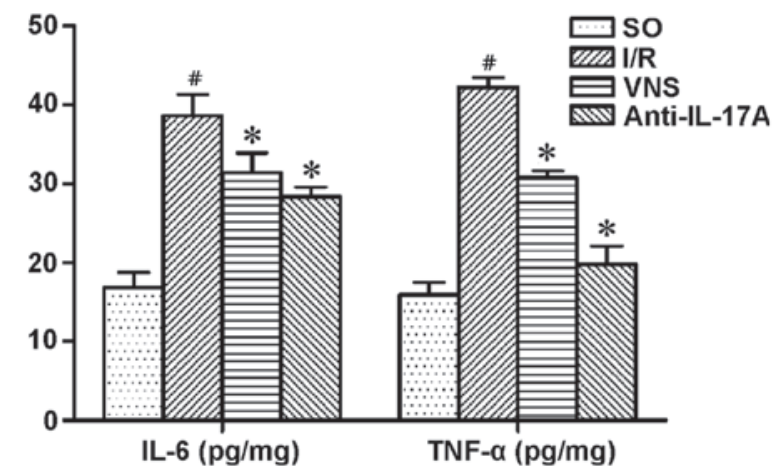

Figure 3. Effects of VNS and anti-IL-17A treatment on TNF- $\alpha$ and IL-6 expression levels during $\mathrm{I} / \mathrm{R}(\mathrm{n}=12$ per group). Data are presented as the mean \pm standard deviation. ${ }^{*} \mathrm{P}<0.05$ vs. the $\mathrm{I} / \mathrm{R}$ group; ${ }^{\#} \mathrm{P}<0.05$ vs. the SO group. VNS, vagal nerve stimulation; I/R, ischemia/reperfusion; anti-IL-17A, anti-interleukin-17A monoclonal antibodies; SO, sham operated control; TNF, tumor necrosis factor; IL, interleukin.

as compared with the other groups $(\mathrm{P}<0.05$; Fig. 3). IL-17A and HMGB1 expression levels in the I/R group were markedly increased, as compared with the other groups. Similarly, 


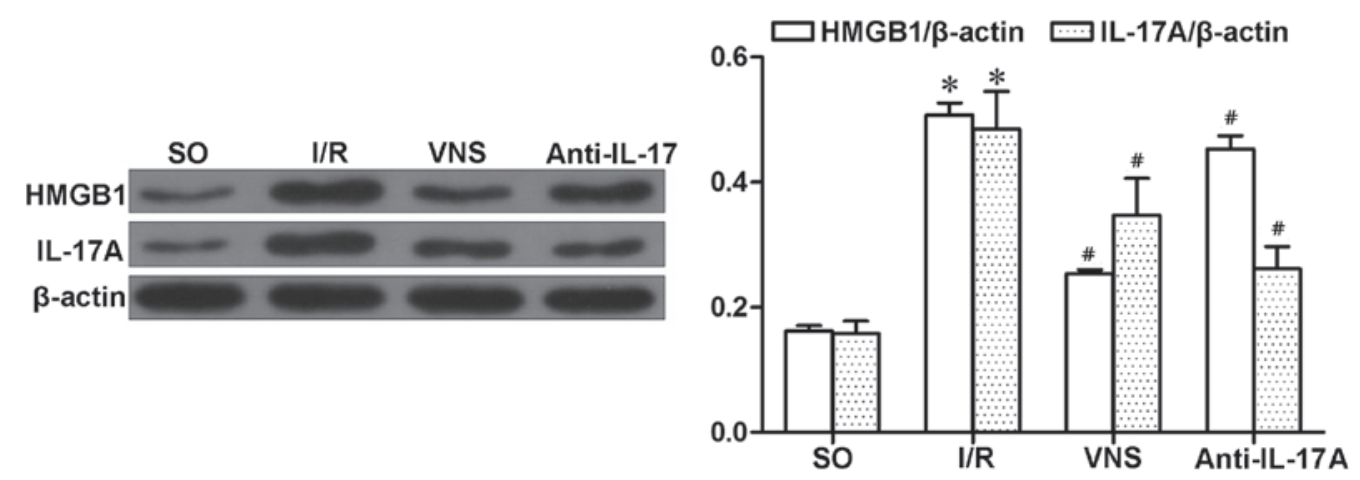

Figure 4. Effects of VNS or anti-IL-17A treatment on HMGB1 and IL-17A expression during I/R ( $\mathrm{n}=12)$. Data are presented as the mean \pm standard deviation. ${ }^{*} \mathrm{P}<0.05$ vs. the $\mathrm{SO}$ group; ${ }^{\#} \mathrm{P}<0.05$ vs. the I/R group. VNS, vagal nerve stimulation; anti-IL-17A, anti-interleukin-17A monoclonal antibodies; I/R, ischemia/reperfusion; SO, sham operated control; HMGB1, high mobility group box 1 protein; IL, interleukin.
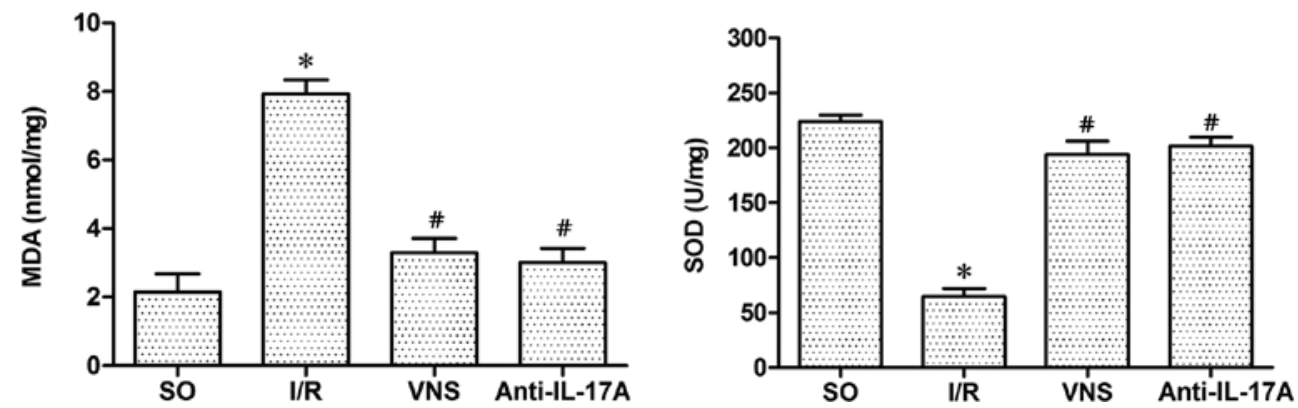

Figure 5. Effects of VNS or anti-IL-17A treatment on MDA and SOD content during I/R ( $\mathrm{n}=12)$. Data are presented as the mean \pm standard deviation." $\mathrm{P}<0.05$ vs. the SO group; ${ }^{\mathrm{P}}<0.05$ vs. the I/R group. MDA, malondialdehyde; SOD, superoxide dismutase; SO, sham operated control; I/R, ischemia/reperfusion; VNS, vagal nerve stimulation; anti-IL-17A, anti-interleukin-17A monoclonal antibodies.
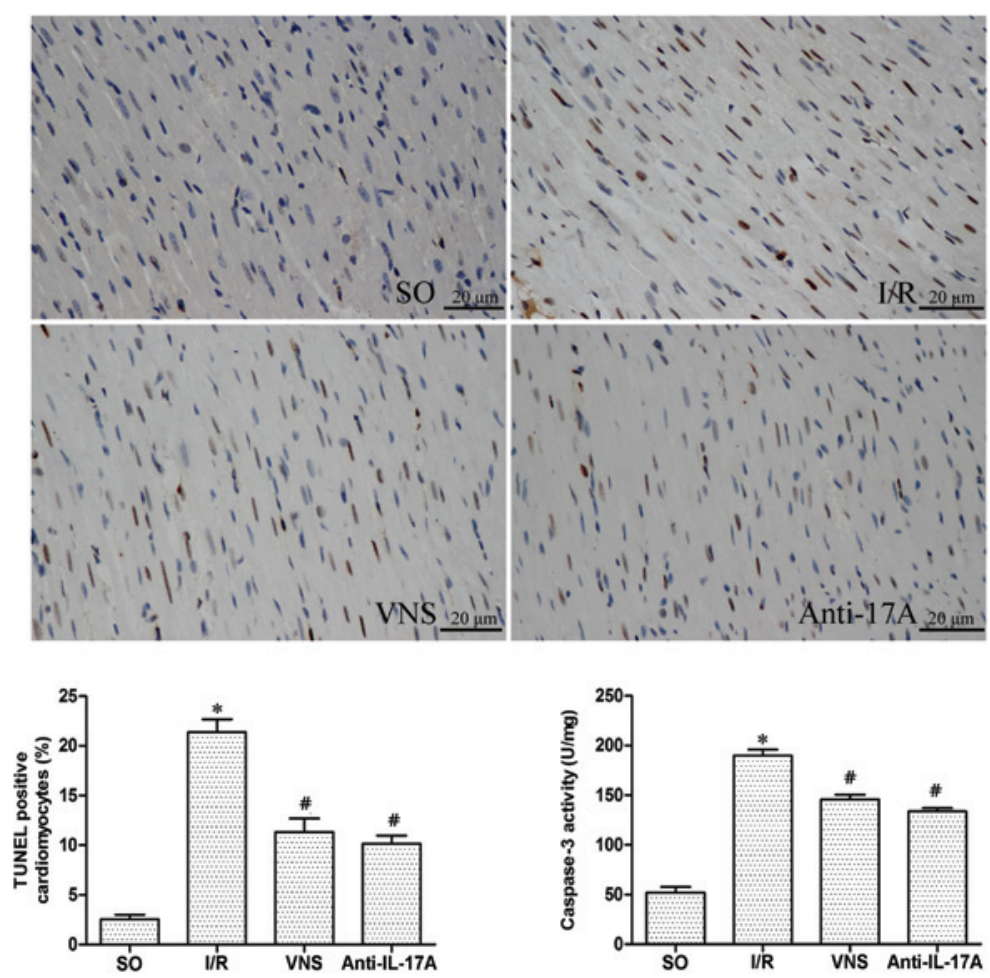

Figure 6. Effects of VNS or anti-IL-17A treatment on the apoptosis of cardiomyocytes during I/R ( $n=12)$. Representative micrographs of TUNEL-stained heart sections from various groups following $4 \mathrm{~h}$ of reperfusion (magnification, $\mathrm{x} 400$ ). Percentage of TUNEL-positive nuclei over total number of nuclei and caspase- 3 activity in the myocardium was assessed following $4 \mathrm{~h}$ of reperfusion. Data are presented as the mean \pm standard deviation. ${ }^{*} \mathrm{P}<0.05$ vs. the SO group; ${ }^{\text {"}} \mathrm{P}<0.05$ vs. the I/R group. SO, sham operated control; TUNEL, terminal deoxynucleotidyl-transferase mediated dUTP nick-end labeling; VNS, vagal nerve stimulation; I/R, ischemia/reperfusion; anti-IL-17A, anti-interleukin-17A mAb. 
VS decreased the expression levels of IL-17A and HMGB1, as compared with the I/R group (both $\mathrm{P}<0.05$ ). Furthermore, administration of anti-IL-17A mAbs inhibited the expression levels of IL-17A and HMGB1 ( $<<0.05$; Fig. 4), which were induced by $\mathrm{I} / \mathrm{R}$ injury. However, the reductions in HMGB1 expression levels were lower ( $\mathrm{P}=0.0318$; Fig. 4), as compared with in IL-17A.

MDA and SOD activity levels. Following I/R, the levels of MDA in the I/R group were significantly increased $(\mathrm{P}<0.05)$, whereas SOD levels were significantly decreased $(\mathrm{P}<0.05)$, as compared with those in the SO group. Conversely, VS or anti-IL-17A administration significantly inhibited the oxidative stress induced by I/R (Fig.5)

Myocardial apoptosis. The percentage of TUNEL-positive cardiomyocytes was markedly increased in the I/R group, as compared with the other treatment groups (Fig. 6). Similar trends in the activity of caspase-3 were demonstrated following I/R. These results suggest that I/R may have induced an increase in the apoptosis of cardiomyocytes, and VS or treatment with anti-IL-17A mAbs may attenuate I/R-induced apoptosis.

\section{Discussion}

Previous studies have demonstrated that vagal activation is capable of exerting cardioprotective effects in various cardiovascular diseases, including ischemic heart disease, heart failure, arrhythmia and hypertension $(7,15)$. In the present study, VS was initiated at 15 min following acute myocardial ischemia and maintained for $30 \mathrm{~min}$, which is similar to a previous study (9). Consistent with previous studies $(14,16)$, VS significantly reduced infarct size following myocardial I/R injury. The results of the present study demonstrated that VS was capable of decreasing myocardial enzyme expression levels and cardiomyocyte apoptosis, which is an important mechanism of cell death. Furthermore, previous studies (16-18) have demonstrated that VS or the neurotransmitter ACh may exert cardioprotective effects through the regulation of mitochondrial biogenesis and function. The prolonged opening of the mitochondrial permeability transition pore induces cell death through the rapid depletion of ATP and activation of death messengers, such as caspases, which have a key role in apoptosis. The present study demonstrated that VS or anti-IL-17A $\mathrm{mAb}$ treatment may regulate the apoptosis of cardiomyocytes, as confirmed by the alterations in TUNEL-positive cardiomyocytes and caspase-3 activity levels. These results suggested that VS may attenuate myocardial I/R injury by restraining myocardial apoptosis via IL-17A, which is a regulator associated with apoptosis.

Inflammation is one of the major pathophysiological mechanisms associated with myocardial I/R injury (19). A previous study demonstrated that the cardioprotective effects of VS in myocardial I/R injury are associated with the activation of the a-7 nicotinic AChR-mediated cholinergic anti-inflammatory pathway (14). HMGB1 is an integral proinflammatory cytokine in myocardial $\mathrm{I} / \mathrm{R}$ injury that communicates with other cytokines, including TNF- $\alpha$, IL-6 and C-reactive protein (20). Furthermore, $\alpha-7 \mathrm{nAChR}$ activation is capable of initiating the Janus-activated kinase/signal transducers and activators of transcription 3-nuclear factor- $\kappa \mathrm{B}$ cascade, which was associated with a decrease in the levels of proinflammatory cytokines including TNF- $\alpha$, interleukins and HMGB1 (21). Zhu et al (22) demonstrated that both HMGB1 and IL-17A expression levels were significantly increased, and HMGB1 facilitated the release of IL-17A, which induced apoptosis and neutrophil infiltration that further aggravated I/R injury. In the present study, VS was able to significantly attenuate myocardial I/R injury and decrease IL-17A, HMGB1, IL-6 and TNF- $\alpha$ expression levels. Furthermore, neutralization of endogenous IL-17A successfully protected cells against myocardial I/R injury, inducing the same protective effects as VS; however the expression levels of HMGB1 induced by I/R injury were only slightly reduced. These results suggested that VS may have a cardioprotective role in myocardial I/R injury by inhibiting HMGB1 expression levels, thus reducing the expression levels of IL-17A.

Oxidative stress is a major detrimental process associated with the pathogenesis of I/R injury. Ekici et al (23) demonstrated that VS exhibits antioxidant efficacy in the treatment of focal cerebral I/R; therefore VS may be capable of suppressing the generation of reactive oxygen species (ROS) in ischemic heart diseases $(24,25)$. VS may also exert its antioxidant effects by suppressing the prolonged opening of the permeable transition pore which promotes the production of ROS (16). Furthermore, IL-17A facilitated the release of proinflammatory cytokines and ROS, which could lead to neutrophilic inflammation (26). Oxidative stress markers were analyzed in the present study, the results of which demonstrated that VS was capable of reducing the levels of MDA, a ROS, and increasing the levels of SOD, which is a key antioxidant enzyme. Furthermore, neutralization of endogenous IL-17A was also able to suppress I/R-induced ROS production. These results demonstrated that VS may alleviate myocardial I/R injury by suppressing the I/R-induced production of ROS, which may be associated with the suppression of IL-17A expression levels.

In the present study, VS intervention was performed as previously described (9). The time schedule of treatment in the present study may differ from the clinical condition. Furthermore, VS and anti-IL-17A mAb treatment demonstrated antioxidant effects and the ability to inhibit apoptosis in cardiomyocytes; thus suggesting that IL-17A may be a downstream regulator of the cholinergic anti-inflammatory pathway activated by VS. Investigation of the VS-HMGB1-IL-17A axis remains necessary, in order to directly understand the relationship between VS and IL-17A.

In conclusion, the present study demonstrated that, in a rat model of myocardial I/R, VS is capable of modulating inflammatory responses and inhibiting oxidative stress and apoptosis-induced injury. Furthermore, VS may attenuate myocardial I/R injury, which may be associated with the inhibition of IL-17A expression.

\section{Acknowledgements}

The present study was partially supported by a grant from the National Natural Science foundation of China (grant no. 81370308). 


\section{References}

1. Yellon DM and Hausenloy DJ: Myocardial reperfusion injury. N Engl J Med 357: 1121-1135, 2007.

2. Iwakura Y, Nakae S, Saijo $S$ and Ishigame H: The roles of IL-17A in inflammatory immune responses and host defense against pathogens. Immunol Rev 226: 57-79, 2008.

3. Ni J, Hu G, Xiong J, Shen J, Shen J, Yang L, Tang M, Zhao Y, Ying G, Yu G, et al: Involvement of interleukin-17A in pancreatic damage in rat experimental acute necrotizing pancreatitis Inflammation 36: 53-65, 2013.

4. Wang X, Sun R, Wei H and Tian Z: High-mobility group box 1 (HMGB1)-Toll-like receptor (TLR)4-interleukin (IL)-23-IL-17A axis in drug-induced damage-associated lethal hepatitis: Interaction of $\gamma \delta \mathrm{T}$ cells with macrophages. Hepatology 57: 373-384, 2013.

5. $\mathrm{Hu} \mathrm{X}, \mathrm{Xu} \mathrm{W}$ and Jiang H: HMGB1/IL-17A axis: An important mechanism for myocardial ischemia-reperfusion injury. Int $\mathbf{J}$ Cardiol 174: 447-448, 2014

6. Liao YH, Xia N, Zhou SF, Tang TT, Yan XX, Lv BJ, Nie SF, Wang J, Iwakura Y, Xiao H, et al: Interleukin-17A contributes to myocardial ischemia/reperfusion injury by regulating cardiomyocyte apoptosis and neutrophil infiltration. J Am Coll Cardiol 59: 420-429, 2012.

7. Zhao M, Sun L, Liu JJ, Wang H, Miao Y and Zang WJ: Vagal nerve modulation: A promising new therapeutic approach for cardiovascular diseases. Clin Exp Pharmacol Physiol 39: 701-705, 2012

8. De Ferrari GM and Schwartz PJ: Vagus nerve stimulation: from pre-clinical to clinical application: challenges and future directions. Heart Fail Rev 16: 195-203, 2011.

9. Wang Q, Cheng Y, Xue FS, Yuan YJ, Xiong J, Li RP, Liao X and Liu JH: Postconditioning with vagal stimulation attenuates local and systemic inflammatory responses to myocardial ischemia reperfusion injury in rats. Inflamm Res 61: 1273-1282, 2012.

10. Park J, Kang JW and Lee SM: Activation of the cholinergic anti-inflammatory pathway by nicotine attenuates hepatic ischemia/reperfusion injury via heme oxygenase-1 induction. Eur J Pharmacol 707: 61-70, 2013.

11. Tang Q, Li J, Zhu H, Li P, Zou Z and Xiao Y: Hmgb1-IL-23-IL-17-IL-6-Stat3 axis promotes tumor growth in murine models of melanoma. Mediators Inflamm 2013: 713859, 2013.

12. Calvillo L, Vanoli E, Andreoli E, Besana A, Omodeo E, Gnecchi M, Zerbi P, Vago G, Busca G and Schwartz PJ: Vagal stimulation, through its nicotinic action, limits infarct size and the inflammatory response to myocardial ischemia and reperfusion. J Cardiovasc Pharmacol 58: 500-507, 2011.

13. National Research Council; Guide for the Care and Use of Laboratory Animals. National Academies Press (US), Washington (DC), 1996.
14. Hu X, Zhou X, He B, Xu C, Wu L, Cui B, Wen H, Lu Z and Jiang $\mathrm{H}$ : Minocycline protects against myocardial ischemia and reperfusion injury by inhibiting high mobility group box 1 protein in rats. Eur J Pharmacol 638: 84-89, 2010.

15. Thayer JF and Lane RD: The role of vagal function in the risk for cardiovascular disease and mortality. Biol Psychol 74: 224-242, 2007.

16. Shinlapawittayatorn K, Chinda K, Palee S, Surinkaew S, Thunsiri K, Weerateerangkul P, Chattipakorn S, KenKnight BH and Chattipakorn N: Low-amplitude, left vagus nerve stimulation significantly attenuates ventricular dysfunction and infarct size through prevention of mitochondrial dysfunction during acute ischemia-reperfusion injury. Heart Rhythm 10: 1700-1707, 2013.

17. Katare RG, Ando M, Kakinuma Y, Arikawa M, Handa T, Yamasaki F and Sato T: Vagal nerve stimulation prevents reperfusion injury through inhibition of opening of mitochondrial permeability transition pore independent of the bradycardiac effect. J Thorac Cardiovasc Surg 137: 223-231, 2009.

18. Sun L, Zhao M, Yu XJ, Wang H, He X, Liu JK and Zang WJ: Cardioprotection by acetylcholine: A novel mechanism via mitochondrial biogenesis and function involving the PGC-1 $\alpha$ pathway. J Cell Physiol 228: 1238-1248, 2013.

19. Eltzschig HK and Eckle T: Ischemia and reperfusion - from mechanism to translation. Nat Med 17: 1391-1401, 2011.

20. Hu X, Fu W and Jiang H: HMGB1: A potential therapeutic target for myocardial ischemia and reperfusion injury. Int J Cardiol 155: 489, 2012.

21. Marrero MB and Bencherif M: Convergence of alpha 7 nicotinic acetylcholine receptor-activated pathways for anti-apoptosis and anti-inflammation: Central role for JAK2 activation of STAT3 and NF-kappaB. Brain Res 1256: 1-7, 2009.

22. Zhu H, Li J, Wang S, Liu K, Wang L and Huang L: Hmgb1-TLR4-IL-23-IL-17A axis promote ischemia-reperfusion injury in a cardiac transplantation model. Transplantation 95: 1448-1454, 2013

23. Ekici F, Karson A, Dillioglugil MO, Gurol G, Kir HM and Ates N: The effects of vagal nerve stimulation in focal cerebral ischemia and reperfusion model. Turk Neurosurg 23: 451-457, 2013.

24. Tsutsumi T, Ide T, Yamato M, Kudou W, Andou M, Hirooka Y, Utsumi H, Tsutsui $\mathrm{H}$ and Sunagawa K: Modulation of the myocardial redox state by vagal nerve stimulation after experimental myocardial infarction. Cardiovasc Res 77: 713-721, 2008.

25. Kong SS, Liu JJ, Yu XJ, Lu Y and Zang WJ: Protection against ischemia-induced oxidative stress conferred by vagal stimulation in the rat heart: Involvement of the AMPK-PKC pathway. Int J Mol Sci 13: 14311-14325, 2012.

26. Pinart M, Zhang M, Li F, Hussain F, Zhu J, Wiegman C, Ryffel B and Chung KF: IL-17A modulates oxidant stress-induced airway hyperresponsiveness but not emphysema. PLoS One 8: e58452, 2013. 